Internat. J. Math. \& Math. Sci.

Vol. 23, No. 6 (2000) 409-413

S0161171200002222

(C) Hindawi Publishing Corp.

\title{
SOME RESULTS ON MAXIMAL ELEMENTS
}

\section{ANTONIO CARBONE}

(Received 31 December 1998)

ABSTRACT. We prove some results on maximal elements using the KKM-map principle.

Keywords and phrases. Maximal elements, KKM-map principle.

2000 Mathematics Subject Classification. Primary 47H10; Secondary 54H25.

The well-known KKM-map principle has various applications in nonlinear analysis, mathematical economics, applied mathematics, game theory, and minimax theory. The aim of this paper is to prove some results on maximal elements by using the KKM-map principle.

Recall that a binary relation $F$ on a set $C$ is a subset of $C \times C$ or a mapping of $C$ into itself. The notation $y F x$ or $y \in F x$ means $y$ stands in relation $F$ to $x$. A maximal element of $F$ is a point $x$ such that no point $y$ satisfies $y \in F x$, i.e., $F x=\varnothing$. Thus the set of maximal elements is

$$
\{x \in C: F x=\varnothing\}=\bigcap_{x \in C}\left(C \backslash F^{-1} x\right),
$$

where

$$
F^{-1} x=\{y \in C: x \in F y\}
$$

The following well-known theorem is due to Sonnenschein [7] proved by using the KKM-principle.

THEOREM 1. Let $C$ be a compact convex subset of $\mathbb{R}^{n}$ and $F$ a binary relation on $C$ satisfying

(i) $x \notin \operatorname{co} F x$ for all $x \in C$ (co stands for convex hull),

(ii) if $y \in F^{-1} x$, then there exists some $x_{1} \in C$ (possibly $x_{1}=x$ ) such that $y \in$ int $F^{-1} x_{1}$.

Then $F$ has a maximal element.

In [1], Bergstrom proved the following by using selection theorem and fixed point theorem.

THEOREM 2. Let $C$ be a nonempty, compact convex subset of $\mathbb{R}^{n}$ and $F: C \rightarrow 2^{C} a$ preference map satisfying

(i) $x \notin \operatorname{co} F x$ for each $x \in C$,

(ii) $F$ is lower semicontinuous on $C$.

Then $F$ has a maximal element. 
We note that the results on maximal elements are useful in fixed point theory, variational inequalities and complementarity problems (see [2]).

DEFINITION 3. Let $C$ be a nonempty subset of a topological vector space $X$. A map $F: C \rightarrow 2^{X}$ is said to be a KKM-map if $\operatorname{co}\left(x_{1}, x_{2}, \ldots, x_{n}\right) \subseteq \cup_{i=1}^{n} F x_{i}$ for each finite subset $\left\{x_{1}, x_{2}, \ldots, x_{n}\right\}$ of $C$.

THEOREM 4 [3]. Let $C$ be a subset of a Hausdorff topological vector space $X$ and $F: C \rightarrow 2^{X}$ a closed-valued map. If $F x_{0}$ is compact for at least one $x_{0} \in C$, then $\cap_{x \in C} F x \notin \varnothing$.

THEOREM 5 [5]. Let $C$ be a subset of a Hausdorff topological vector space $X$ and $F: C \rightarrow 2^{X}$ an open-valued KKM-map. Then $\cap_{i=1}^{n} F x_{i} \neq \varnothing$.

KY FAN'S LEMMA [3]. Let $C$ be a nonempty compact convex subset of $\mathbb{R}^{n}$ and $F: C \rightarrow$ $2^{C}$ a multifunction such that

(i) Fx is convex for each $x \in C$,

(ii) $F$ has open graph,

(iii) $x \notin F x$ for each $x \in C$.

Then $F$ has a maximal element.

We prove the following theorem for maximal element.

THEOREM 6. Let $C$ be a nonempty closed convex subset of a Hausdorff topological vector space $X$ and $F: C \rightarrow 2^{C}$ satisfy

(i) $x \notin F x$ for each $x \in C$,

(ii) $F x$ is closed for each $x \in C$,

(iii) $F^{-1}(y)=\{x \in C: y \in F x\}$ is convex for each $y \in C$,

(iv) $C$ can be covered by some finite number of closed sets $F x_{1}, F x_{2}, \ldots, F x_{n}$.

Then $F$ has a maximal element.

Proof. Let $F x \neq \varnothing$ for each $x \in C$. Define $G(x)=C \backslash F x$, then $G(x)$ is open for each $x \in C$ since $F x$ is closed. By (iv) $C=\cup_{i=1}^{n} F x_{i}$, so $\cap G x_{i}=\cap\left(C \backslash F x_{i}\right)^{\mathrm{c}}=\left(\cup F x_{i}\right)^{\mathrm{c}}=\varnothing$. Therefore $G$ is not a KKM-map. Hence, there exists a finite set $\left\{x_{1}, x_{2}, \ldots, x_{k}\right\}$ of $C$ such that

$$
z=\sum_{i=1}^{k} \lambda_{i} x_{i} \not \subset \bigcup_{1}^{k} G x_{i},
$$

where $\lambda_{i} \geq 0$ and $\sum \lambda_{i}=1$. So $z \in \cap_{i=1}^{n} F x_{i}$ and $x_{i} \in F^{-1} z, i=1,2, \ldots, k$. Since $F^{-1} z$ is convex so

$$
z=\sum_{i=1}^{k} \lambda_{i} x_{i} \in F^{-1} z,
$$

implying that $z \in F z$, this contradicts hypothesis (i). So $F x=\varnothing$.

COROLLARY 7. Let $C$ be a nonempty closed convex subset of a topological vector space $X$ and $F: C \rightarrow 2^{C}$ an upper semicontinuous convex-valued map. Further, assume that there is some finite subset $B$ of $C$ such that $F x \cap B \neq \varnothing$ for every $x \in C$, and $x \notin F x$ for each $x \in C$. Then $F$ has a maximal element. 
Proof. Let $G: C \rightarrow 2^{C}$ be defined by

$$
G x=F^{-1} x=\{y \in C: x \in F y\} .
$$

Since $F$ is upper semicontinuous, each $G(x)$ is closed. Now, $G^{-1}(y)=\left(F^{-1} y\right)^{-1}=$ $F y$ is convex. Also $C$ can be covered by $\{G(x): x \in B\}$, finitely many closed sets. By hypothesis $x \notin F x$ For $x \in C$. So, by Theorem 5 , we get that $F$ has a maximal element.

In the following, closed-valued KKM-map is applied [6].

THEOREM 8. Let $C$ be a nonempty convex subset of a topological vector space $X$, and $F: C \rightarrow 2^{C}$ satisfy

(i) $x \notin \operatorname{co} F x$ for each $x \in C$,

(ii) if $y \in F^{-1} x$, then there exists some $x_{1} \in C$ (possibly $x_{1}=x$ ) such that $y \in \operatorname{int} F^{-1} x_{1}$,

(iii) $C$ has a nonempty compact convex subset $D$ such that the set

$$
B=\{x \in C: y \notin F x \text { for all } y \in D\}
$$

is compact.

Then $F$ has a maximal element.

Proof. Define $G(x)=C \backslash \operatorname{int} F^{-1}(x)$ for each $x \in C$. Then $G(x)$ is closed for each $x \in C$. We claim that $G$ is a KKM-map. Let $z \in \operatorname{co}\left(x_{1}, x_{2}, \ldots, x_{n}\right)$. If $z \notin \cup_{i=1}^{n} G x_{i}$, then $z \notin G x_{i}, i=1,2, \ldots, n$, that is, $z \in F^{-1} x_{i}, i=1,2, \ldots, n$. Thus $x_{i} \in F z$ and $z \in \operatorname{co} F z$, this contradicts (i). Hence $G$ is a KKM-map.

Now,

$$
\begin{aligned}
B & =\{x \in C: y \notin F x \text { for all } y \in D\} \\
& =\left\{x \in C: x \notin F^{-1} y \text { for all } y \in D\right\} \\
& =\{x \in C: x \in G y \text { for all } y \in D\} \\
& =\bigcap_{y \in D} G y=\varnothing,
\end{aligned}
$$

i.e., $x_{0} \in \bigcap_{y \in D} G y$. Thus $x_{0} \notin F^{-1} y$ for all $y \in C$, that is, $y \notin F x_{0}$ for all $y \in C$ and $F x_{0}=\varnothing$.

COROLLARY 9. Let $C$ be a nonempty convex subset of a topological vector space $E$ and $F: C \rightarrow 2^{C}$ satisfy

(i) $x \notin F x$,

(ii) $F x$ is convex for each $x \in C$,

(iii) $F^{-1}(y)=\{x \in C: y \in F x\}$ is open in $C$ for each $y \in C$,

(iv) $C$ has a nonempty compact convex subset $D$ such that the set

$$
B=\{x \in C: y \notin F x \text { for all } y \in D\}
$$

is compact.

Then $F$ has a maximal element. 
COROLLARY 10 (see [6]). Let $C$ be a nonempty convex subset of a topological vector space $X$ and $F: C \rightarrow 2^{C}$ satisfy

(i) $x \notin \operatorname{co} F(x)$ for each $x \in C$

(ii) $F^{-1}(y)=\{x \in C: y \in F x\}$ is open for each $y \in C$,

(iii) $C$ has a nonempty compact convex subset $D$ such that the set

$$
B=\{x \in C: y \notin F x \text { for all } y \in D\}
$$

is compact.

Then $F$ has a maximal element.

In case $C$ is a nonempty compact convex subset of a topological vector space $X$ and $F: C \rightarrow 2^{C}$ satisfy

(i) $x \notin \operatorname{co} F(x)$ for each $x \in C$,

(ii) $F^{-1}(y)=\{x \in C: y \in F x\}$ is open for each $y \in C$,

then $F$ has a maximal element.

We conclude by giving a couple of applications of maximal elements.

THEOREM 11 (Ky Fan best approximation theorem [4]). Let $C$ be a compact, convex subset of $\mathbb{R}^{n}$ and $f: C \rightarrow R^{n}$ a continuous function. Then there is an $x_{0} \in C$ such that

$$
\left\|f x_{0}-x_{0}\right\| \leq\left\|f x_{0}-y\right\| \text { for all } y \in C \text {. }
$$

Proof. Define $F$ by $y F x$ if and only if

$$
\|f x-y\|<\|f x-x\| \text { for all } x \in C \text {. }
$$

It is easy to see that $F x$ is convex and $x \notin F x$. $F$ has open graph because $f$ is continuous. Therefore by Fan's lemma, $F$ has a maximal element, that is, there is an

$$
\bar{x} \in C
$$

such that $F \bar{x}=\varnothing$. This implies that $\|f \bar{x}-\bar{x}\| \leq\|f \bar{x}-y\|$ for all $y \in C$.

NotE. In case $f: C \rightarrow C$, then $f$ has fixed point, that is, there is an $x_{0} \in C$ such that $f x_{0}=x_{0}$.

The following result deals with the existence of zero for a continuous function.

THEOREM 12. Let $C$ be a closed bounded convex subset of $\mathbb{R}$ and $g: C \rightarrow \mathbb{R}$ a continuous function. If $f x=g x+x$ and $f: C \rightarrow C$, then there is an $x_{0} \in C$ such that $g x_{0}=0$.

Proof. Define $F$ on $C$ by $y \in F x$ if and only if $|f x-y|<|f x-x|$ for each $x \in C$. As in the above theorem we get $F x_{0}=\varnothing$. For some $x_{0} \in C$, that is, $\left|f x_{0}-x_{0}\right| \leq\left|f x_{0}-y\right|$ for all $y \in C$. Thus $\left|f x_{0}-x_{0}\right|=d\left(f x_{0}, C\right)$. Since $f: C \rightarrow C$ so $f x_{0}=x_{0}=g x_{0}+x_{0}$. Hence $g x_{0}=0$.

Similar results can be proved by the use of maximal elements. 


\section{REFERENCES}

[1] T. Bergstrom, The Existence of Maximal Elements and Equilibria in the Absence of Transitivity, University of Michigan, 1975, Lecture note.

[2] K. C. Border, Fixed point theorems with applications to economics and game theory, Cambridge University Press, Cambridge, 1985. MR 86j:90002. Zbl 558.47038.

[3] K. Fan, A generalization of Tychonoff's fixed point theorem, Math. Ann. 142 (1961), 305310. MR 24\#A1120. Zbl 093.36701.

[4] Ex _ Extensions of two fixed point theorems of F. E. Browder, Math. Z. 112 (1969), 234240. MR 40\#4830. Zbl 185.39503.

[5] W. K. Kim, Some applications of the Kakutani fixed point theorem, J. Math. Anal. Appl. 121 (1987), no. 1, 119-122. MR 88h:55002. Zbl 612.54055.

[6] T. C. Lin, Convex sets, fixed points, variational and minimax inequalities, Bull. Austral. Math. Soc. 34 (1986), no. 1, 107-117. MR 87h:47128. Zbl 597.47038.

[7] H. F. Sonnenschein, Demand theory without transitive preferences, with applications to the theory of competitive equilibrium, Preferences, utility and demand (a Minnesota symposium) (New York), Harcourt Brace Jovanovich, 1971, pp. 215-223. MR 56\#17746. Zbl 277.90012.

Carbone: Dipartimento di Matematica, Università della Calabria, 87036 ArCavaCATA DI RENDE (COSENZA), ITALY

E-mail address: carbonea@unica1.it 


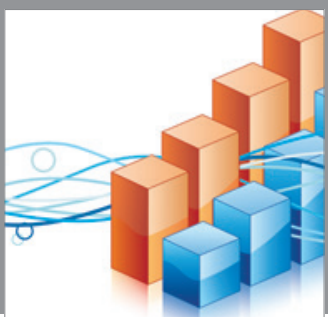

Advances in

Operations Research

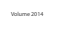

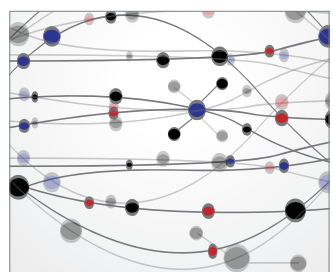

\section{The Scientific} World Journal
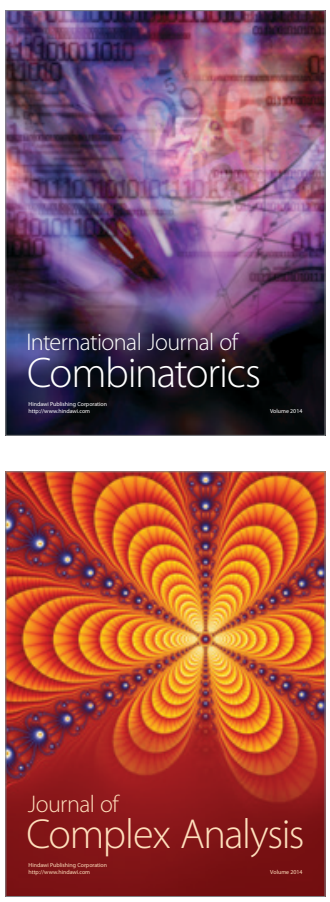

International Journal of

Mathematics and

Mathematical

Sciences
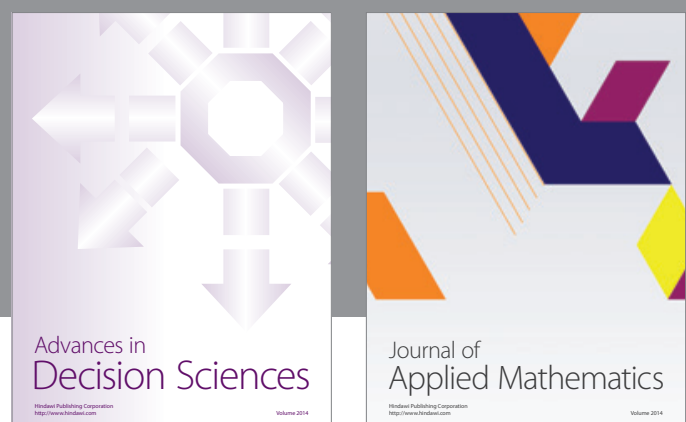

Journal of

Applied Mathematics
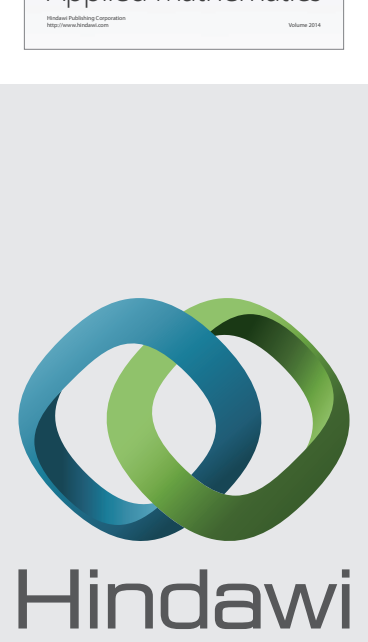

Submit your manuscripts at http://www.hindawi.com
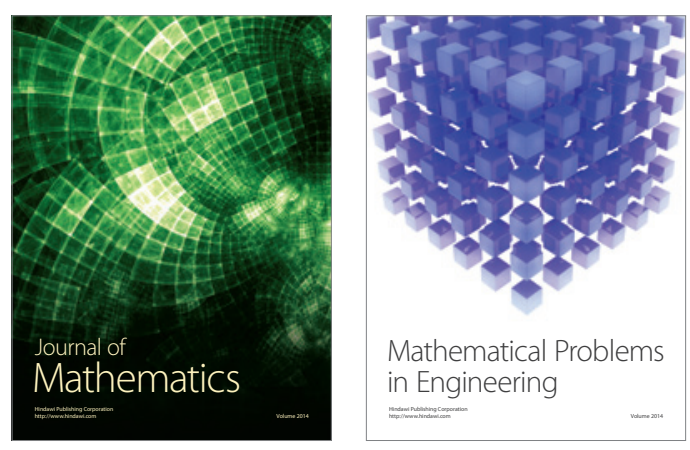

Mathematical Problems in Engineering
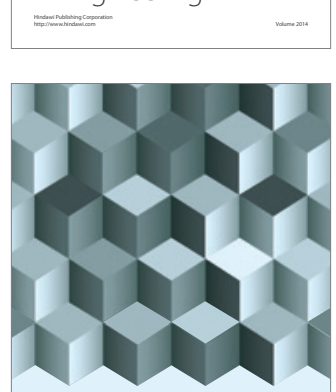

Journal of

Function Spaces
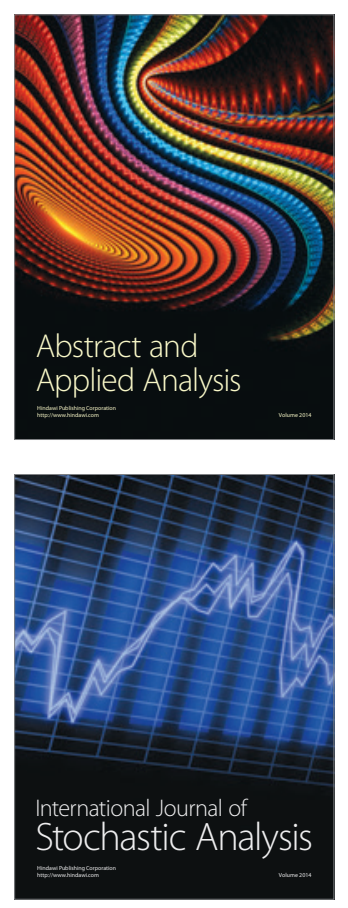

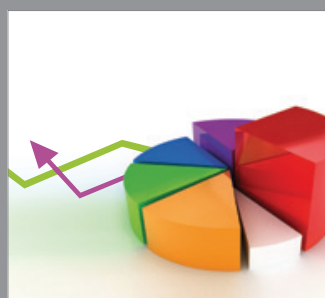

ournal of

Probability and Statistics

Promensencen
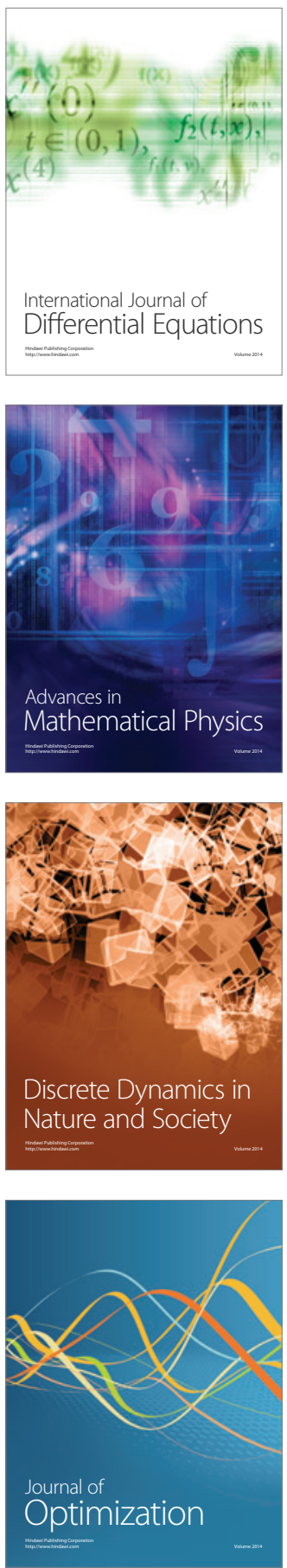